Herz 2017· 42:793

DOI 10.1007/s00059-016-4516-3

Received: 13 November 2016

Accepted: 18 November 2016

Published online: 15 December 2016

C Springer Medizin Verlag Berlin 2016

CrossMark

Kundi et al. [1] reported that the ratio of monocyte to high-density lipoprotein cholesterol (MHR) levels was significantly higher in patients with stable coronary artery disease (SCAD) and a high SYNTAX score. MHR is a simple and widely available marker and they recommended the use of MHR as a prognostic marker for SCAD patients. We thank the authors for their contribution.

It has been demonstrated that inflammation and oxidative stress play a significant role in the formation and progression of atherosclerosis [2]. Circulating monocytes interact with platelets and endothelial cells thereby leading to aggravation of inflammatory and prothrombotic pathways [3]. High-density lipoprotein (HDL) inhibits the migration of macrophages, deactivates the pro-inflammatory and pro-oxidant effects of monocytes, and defuses oxidized LDL molecules, as well as promoting the efflux of cholesterol from these cells [4]. Consequently, MHR was defined as an easily available inflammatory and oxidative stress marker.

MHR has been reported as a new prognostic marker in cardiovascular diseases [5, 6]. Canbolat et al. [7] reported that a higher MHR, which indicates an enhanced inflammation and oxidative stress, was significantly and independently associated with the presence of slow coronary flow. In a recent study by Ucar, both MHR and uric acid (UA) emerged as significant independent predictors of bare metal stent restenosis [8].

There are many biomarkers of inflammation, but it is difficult to measure oxidative stress in vivo. Easily available and well-known markers of oxidative stress include UA and gamma-glutamyl transferase (GGT) $[9,10]$. In this wellpresented article by Kundi and col-

\title{
Selami Soylemez
}

Department of Cardiology, Kadirli State Hospital, Kadirli/Osmaniye, Turkey

\section{Oxidative stress and Syntax score}

leagues, MHR was significantly higher in patients in the high SYNTAX score group compared with the low SYNTAX score group. Additionally C-reactive protein levels were significantly higher in the high SYNTAX score group. On the other hand, the serum UA and GGT levels of the study patients were not reported. It may be beneficial to know the UA and GGT values in order to assess the relationship between oxidative stress and SYNTAX score in patients with SCAD.

\section{Corresponding address}

\section{S. Soylemez, MD}

Department of Cardiology, Kadirli State Hospital

Turgut Ozal Street, 80750 Kadirli/Osmaniye, Turkey

drsoylemez@hotmail.com

Conflict of interest. S. Soylemez declares that he has no competing interests.

\section{References}

1. Kundi $H$, Kiziltunc E, Cetin M, Cicekcioglu $H$, Cetin ZG, CicekGetal (2016) Association monocyte/HDL$C$ ratio with SYNTAX scores in patients with stable coronary artery disease. Herz 41(6):523-529

2. Hansson GK (2005) Inflammation, atherosclerosis, and coronary artery disease. $\mathrm{N}$ Engl J Med 352(16):1685-1695

3. Ancuta P, Wang J, Gabuzda D (2006) CD16+ monocytes produce IL- $6, \mathrm{CCL} 2$, and matrix metalloproteinase- 9 upon interaction with $\mathrm{CX} 3 \mathrm{CL} 1$ expressing endothelial cells. J Leukoc Biol 80:1156-1164

4. Parthasarathy S, Barnett J, Fong LG (1990) High-density lipoprotein inhibits the oxidative modification of low-density lipoprotein. Biochim Biophys Acta 1044:275-283

5. Akboga MK, Yayla C, Balci KG, Ozeke O, Maden O, Kisacik H et al (2016) Relationship between serum albumin level and monocyte-to-high-density lipoprotein cholesterol ratio with saphenous vein graft disease in coronary bypass. Thorac Cardiovasc Surg. doi:10.1055/s-0036-1582260

6. Cetin EH, Cetin MS, Canpolat U, Aydin S, Topaloglu S, Aras D et al (2015) Monocyte/HDL-cholesterol ratio predicts the definite stent thrombosis after primary percutaneous coronary intervention for ST-segment elevation myocardial infarction. BiomarkMed 9(10):967-977

7. Canpolat U, Çetin EH, Cetin S, Aydin S, Akboga MK, Yayla C et al (2016) Association of monocyte-to$\mathrm{HDL}$ cholesterol ratio with slow coronary flow is linked to systemic inflammation. Clin Appl Thromb Hemost 22(5):476-482

8. Ucar FM (2016) A potential marker of bare metal stent restenosis: monocyte count - to- HDL cholesterol ratio. BMC Cardiovasc Disord 16(1):186

9. Feig DI, Kang DH, Johnson RJ (2008) Uric acid and cardiovascular risk. N Engl J Med 359:1811-1821

10. Mason JE, Starke RD, Van Kirk JE (2010) Gammaglutamyl transferase: a novel cardiovascular risk biomarker. Prev Cardiol 13:36-41 